laser. The cluster/particle beam is injected into a high vacuum chamber and ionized using an excimer laser for time-of-flight mass spectroscopy. A beam chopper running synchronously with the $\mathrm{CO}_{2}$ laser is used for size selection. High-resolution electron microscopy was used to reveal details of the crystalline structure of silicon nanoparticles as a function of size. Huisken also described work on photoluminescence of the nanoparticles. Size selection is important for photoluminescence, and smaller nanoparticles result in a wider bandgap. A uv laser was used to excite the films and the photoluminescence was spectrally analyzed. Larger particles were seen to luminesce at a lower energy, or longer wavelength. An astrophysical connection was made in this session: A broad red emission band is observed in astronomical objects, for example in the Orion constellation. This has been attributed to photoluminescence of an interstellar dust grain component excited by the uv radiation from a nearby star. This astrophysical aspect was discussed also in a separate paper by $O$. Guillois (CEA, France) in Symposium I.

Computational materials science, the topic of Symposium C, is showing a closer integration than before of the diverse methodologies and progress toward hybrid methods. To study electronic structure and dynamics simultaneously, the Car-Parrinello technique has become a real "workhorse" and it is the method of choice for systems of up to a few hundred atoms observed over time periods on the order of 10 ps. For larger systems or longer times, tight-binding molecular dynamics is the preferred approach. Several scientists reported applications of these methods to novel fields such as biomolecules and pharmacological systems, besides the more traditional materials (e.g., metals and semiconductors). Classical electronic band structure methods continue to be very useful tools yielding a great deal of information, from binding energies and activation barriers to excited states.

Classical particle-based simulations, using the Monte Carlo (MC) or molecular dynamics (MD) approaches, increasingly rely on energetic information obtained from first-principles calculations rather than resorting to a phenomenological description. With continuing advances in computer power, MC and MD now permit the study of mesoscopic systems and in some cases approach the scale of macroscopic objects. The largest system reported at the Symposium had over $5,000,000$ atoms, with time-scales in MD simulations approaching milliseconds (on the order of tens of millions of time-steps). Spectacular though these numbers may be, only continuum methods permit stud- ies of truly macroscopically large systems, over long time scales (in some cases ranging on the order of centuries). Impressive work along those lines was reported for electromigration, metallic foams, and micromagnetism, to name a few. Here too, major progress has been made in the last few years.

Clearly the main challenge in the years ahead will be the integration of the various methods to span time and length scales. Some work in this direction was presented

at the meeting and showed very encouraging signs that major breakthroughs can be expected in this direction in the next few years. There was also strong evidence of the continuing efforts to use the tools of computational materials science to study "real" materials: systems with imperfections or highly complex unit cells, in high-energy collisions, or interacting with solvents.

Reported by G. R. Rao with contributions from E-MRS symposium organizers.

\title{
MRS-I Announces XIth AGM
}

The XIth Annual General Meeting (AGM) of the Materials Research Society of India (MRS-I) will be held in Baroda on February $3-5,2000$. It is being organized by the Gujarat Chapter of MRS-I, Sardar Patel University, and Indian Petrochemical Corporation.

The XIth AGM will feature a Theme Seminar on Materials in the Service of Chemical Industries with special emphasis on the materials for the new century.
MRS-I Medal recipients will present lectures in the areas of biomaterials, ceramics and glasses, electronic materials, metals, processing technologies, materials characterization, building materials, composites, polymers, magnetic materials, computeraided design of materials, thin films, and fracture. See Calendar listing on page 85.

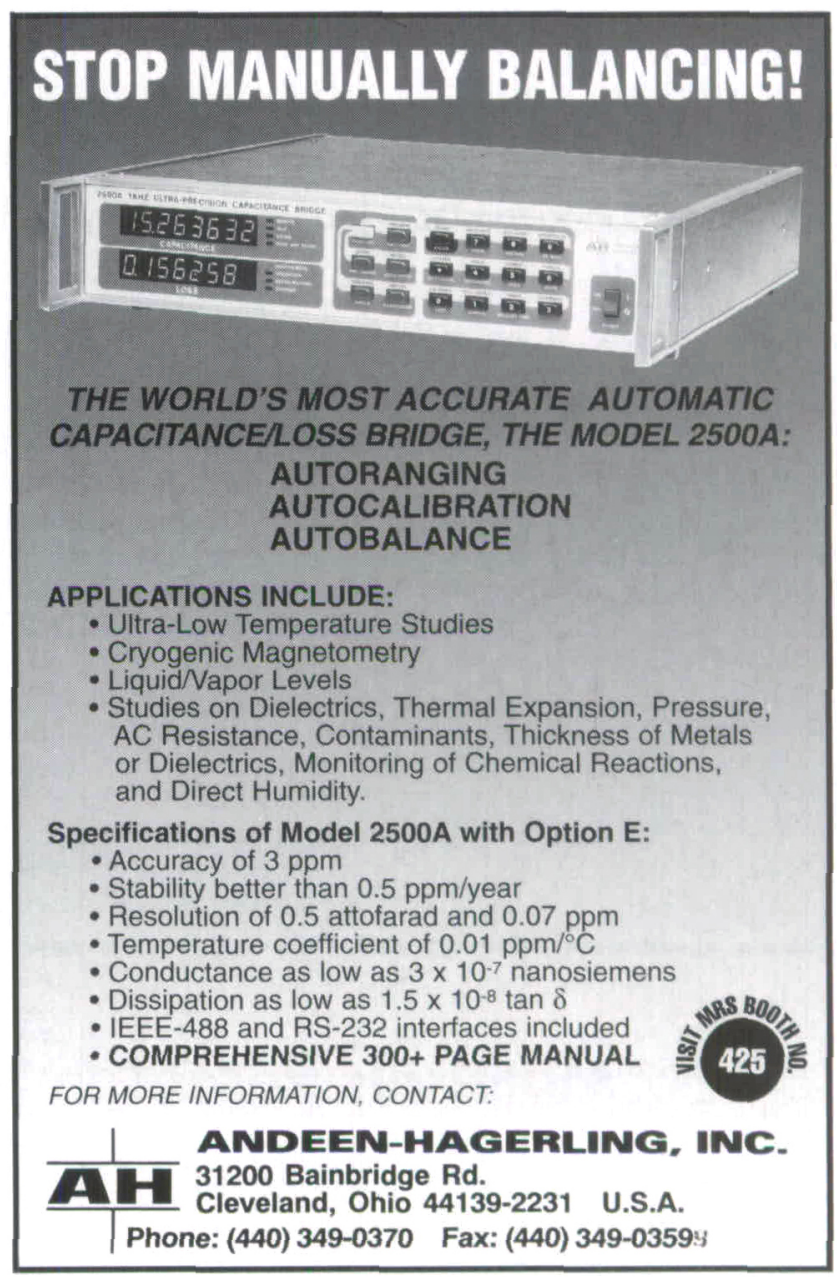

Circle No. 6 on Reader Service Card. 